\section{A case of pseudo-Terson's syndrome}

Terson's syndrome is characterised by vitreous haemorrhage in association with subarachnoid haemorrhage. We report a case of pseudo-Terson's syndrome. Complete resolution of optic disc neovascularisation was observed with medical treatment of intermediate uveitis.

\section{Case report}

A 22-year-old woman presented with sudden loss of vision in the right eye associated with a 2 month history of increasing headache. The following day the left eye became similarly blind, reducing the visual acuity to hand movements in both.

Examination initially revealed bilateral vitreous haemorrhage. She was suspected of having bilateral vitreous haemorrhage secondary to subarachnoid haemorrhage (Terson's syndrome). CT scanning, lumbar puncture and cerebral angiography showed no abnormality. The vitreous haemorrhage gradually cleared and optic disc neovascularisation was revealed. On further questioning she admitted to a history of intermittent episodes of black dots and floaters causing haziness of vision.

Visual acuity was now $6 / 6$ in the right and $6 / 9$ in the left. There was a low-grade vitritis with some vitreous condensation inferiorly. Active neovascularisation was observed at both optic discs (Fig. 1). Physical examination was normal with no neurological deficit or evidence of Takayasu's arteritis. Fluorescein angiography showed some areas of capillary drop-out at the posterior pole with microaneurysm formation. There was marked leakage from the new vessels.

Full blood count, biochemical profile, autoantibodies, immunoglobulins, angiotensin converting enzyme, complement, electrocardiogram and chest radiograph were all normal. ESR was $2 \mathrm{~mm} / \mathrm{h}$. Anti-cardiolipins G, $A$ and $M$ were negative. Protein $C, S$ and anti-thrombin III levels were normal. Factor V Leyden mutation assay was negative. FACS analysis showed no evidence of systemic T-cell activation. Carotid Doppler examination showed normal flow patterns bilaterally. Ultrasound biomicroscopy confirmed the clinical impression of very low-grade vitritis in the region of the pars plana.

A diagnosis of intermediate uveitis with secondary neovascularisation was made. Treatment was initiated with prednisolone $40 \mathrm{mg}$ daily, cyclosporin $125 \mathrm{mg}$ b.d. and ranitidine $150 \mathrm{mg}$ b.d. The prednisolone was gradually reduced over 4 weeks to a maintenance dose of $15 \mathrm{mg}$ daily. Review at this time showed regression of the new vessels with vision of $6 / 6$ N5 in the right and 6/9 N5 in the left. Unfortunately the patient developed significant hypertrichosis as a side-effect of the cyclosporin although this improved with reduction of the dose to $75 \mathrm{mg}$ b.d.

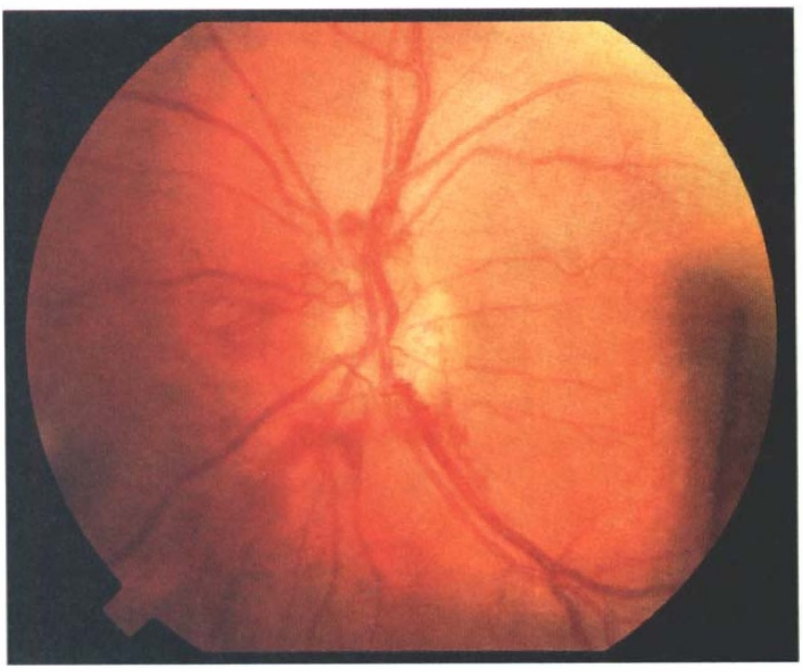

Fig. 1. Neovascularisation at the left optic disc.

The patient is currently maintained on cyclosporin 75 $\mathrm{mg}$ b.d. and prednisolone $7 \mathrm{mg}$ daily. Visual acuities are stable with no evidence of recurrent neovascularisation.

\section{Comment}

Unilateral and bilateral retinal haemorrhages are a common finding in subarachnoid haemorrhage, with venous stasis secondary to extravasated blood in the cerebrospinal fluid the likely pathogenesis. If the haemorrhage is large enough it is liable to break through the internal limiting membrane into the vitreous. Terson's syndrome was named after the ophthalmologist who first described the association of vitreous haemorrhage and subarachnoid haemorrhage in 1927.

Intermediate uveitis is the anatomical term recommended for the description of intraocular inflammation occurring around the posterior ciliary body, pars plana and peripheral retina. It is suggested that this term should replace descriptions such as pars planitis, posterior cyclitis, hyalitis and basal retinochoroiditis. ${ }^{1}$ The pathogenesis remains unclear despite the first description of the disease being almost 50 years ago, ${ }^{2}$ although systemic associations with demyelinating disease and sarcoidosis have been reported. ${ }^{3}$

The clinical manifestations of intermediate uveitis may be very diverse. It tends to be a disease of young adulthood, often with bilateral involvement. The typical presentation is of bilateral chronic vitritis with minimal anterior chamber signs. Reported complications include cataract, cystoid macular oedema, epiretinal membrane, retinal detachment and retinal neovascularisation. ${ }^{4}$ Boke $^{5}$ has further classified intermediate uveitis into three types: diffuse inflammatory, exudative and vasoproliferative characterised by vascular occlusion and neovascularisation. Different patterns of neovascularisation have been described, with new vessels in the peripheral retina more common than disc involvement. ${ }^{6}$ 
Two factors are likely to be responsible for this patient's disc new vessels. The fluorescein angiogram suggested retinal ischaemia due to retinal vasculitis. Hypoxia stimulates the production of vascular endothelial growth factor (VEGF) by retinal cells as a prelude to overt neovascularisation. ${ }^{7}$

Inflammatory processes can also induce new vessel formation independent of ischaemic vasculitis. The most potent mediators are thought to be metabolites of arachidonic acid, in particular prostaglandins $\mathrm{E}_{1}$ and $\mathrm{E}_{2} .{ }^{8,9}$ Cyclo-oxygenase inhibitors, which inhibit the production of these prostaglandins, reduce new vessel formation. ${ }^{10,11}$

Steroids and cyclosporin decrease the liberation of inflammatory mediators and subsequently improve retinal perfusion, inhibiting ischaemic and prostaglandin-induced neovascularisation. Furthermore, it has been recognised for some time that corticosteroids have an anti-angiogenic effect in active neovascularisation which appears to be independent of their anti-inflammatory activity. ${ }^{12}$

In summary, bilateral vitreous haemorrhage and headache is not pathognomonic of Terson's syndrome. It is suggested that a thorough search for underlying ocular inflammatory disease should be made in cases of apparently 'idiopathic' retinal neovascularisation. Ideally assessment should include fluorescein angiography and ultrasound biomicroscopy where available.

\section{References}

1. Bloch-Michel E, Nussenblatt RB. International uveitis study group recommendations for the evaluation of intraocular inflammatory disease. Am J Ophthalmol 1987;103:234-5.

2. Schepens CL. L'inflammation de la region de l'ora serrata et ses sequelles. Bull Mem Soc Fr Ophtalmol 1950;63:113-25.

3. Dinning WJ. Intermediate uveitis: history, terminology, definition. Pars planitis: systemic disease associations. Dev Ophthalmol 1992;23:3-8.

4. Malinowski SM, Pulido JS, Folk JC. Long-term visual outcome and complications associated with pars planitis. Ophthalmology 1993;100:818-24.

5 . Boke W. Clinical picture of intermediate uveitis. Dev Ophthalmol 1992;23:20-7.

6. Felder KS, Brockhurst RJ. Neovascular fundus abnormalities in peripheral uveitis. Arch Ophthalmol 1982;100:750-4.

7. Pe'er J, Schweiki D, Itin, A, et al. Hypoxia induced expression of vascular endothelial growth factor by retinal cells is a common factor in neovascularising ocular diseases. Lab Invest 1995;72:638-45.

8. Ziche M, Jones J, Gullino PM. Role of prostaglandin $\mathrm{E}_{1}$ and copper in angiogenesis. J Natl Cancer Inst 1982;69:475-82.

9. Form DM, Auerbach R. $\mathrm{PGE}_{2}$ and angiogenesis. Proc Soc Exp Biol Med 1982;172:214-8.

10. Frucht J, Zauberman H. Topical indomethacin effect on neovascularisation of the cornea and on prostaglandin $\mathrm{E}_{2}$ levels. Br J Ophthalmol 1984;68:656-8.

11. Haynes WL, Proia AD, Klintworth GK. Effect of inhibitors of arachidonic acid metabolism on corneal vascularisation in the rat. Invest Ophthalmol Vis Sci 1989;30:1588-93.

12. Folkman J, Ingber DE. Angiostatic steroids: method of discovery and mechanism of action. Ann Surg 1987;206:374.

A.T. Purdie

J.A. Olson

The Eye Clinic
Aberdeen Royal Infirmary

Aberdeen, UK

Dr A.T. Purdie

The Eye Clinic

Aberdeen Royal Infirmary

Foresterhill

Aberdeen AB9 2DY, UKUK

Sir,

\section{Paroxetine and acute angle-closure glaucoma}

Acute angle-closure glaucoma has been associated with tricyclic antidepressants and monoamine oxidase inhibitors. In susceptible patients it would appear sensible to use an anti-depressant with weak anticholinergic activity and the use of selective serotonin re-uptake inhibitors (SSRIs) - now widely prescribed seems an appropriate choice of drug. However, paroxetine (an SSRI) has been implicated recently in three separate case reports of acute angle-closure glaucoma. ${ }^{1-3}$ We report a further case of unilateral acute angle-closure glaucoma related to paroxetine.

\section{Case report}

A 53-year-old woman attended the eye clinic with a history of left ocular pain, browache and blurred vision for 1 day. There was congestion of the conjunctiva, shallow anterior chamber and the pupil was mid-dilated with a high intraocular pressure of $61 \mathrm{mmHg}$ on Goldmann tonometry (normal value $\leqslant 21 \mathrm{mmHg}$ ). The patient's spectacle prescription was $[R]+6.50 /+1.00160$ and $[\mathrm{L}]+7.00 /+1.0030$; she had worn glasses for hypermetropia since the age of 5 years. A diagnosis of acute angle-closure glaucoma was made and she was admitted for standard medical treatment. Nd:Yag laser peripheral iridotomies were performed to both eyes and she made an unremarkable recovery with normal intraocular pressure measurements off all topical treatment. A similar, but less severe episode had occurred 6 months previously when she had been prescribed paroxetine $20 \mathrm{mg}$ once daily. She had stopped the medication herself and the symptoms had resolved spontaneously. Interestingly, she had re-commenced the same medication just 3 days prior to her later presentation with acute angle-closure glaucoma.

\section{Comment}

Incomplete mydriasis is the trigger for acute angleclosure glaucoma in susceptible eyes and hence dim ambient lighting conditions and the use of drugs with anticholinergic or adrenergic properties (e.g. bronchodilators and tricyclic antidepressants) have been implicated in its aetiology. Three previous case reports ${ }^{1-3}$ describe a link between the use of paroxetine and the onset of acute angle-closure glaucoma in patients whose ages ranged from 70 to 91 years. In only one report was the patient's refraction referred to qualitatively as hypermetropic. The patient in our case was a more typical age for suffering an acute angle-closure glaucoma 